\title{
Der Aufbau des Europäischen Auswärtigen Dienstes: Stand und Perspektiven
}

\author{
Julia Lieb und Martin Kremer*
}

Wie kann die Europäische Union nach dem Inkrafttreten des Lissabonner Vertrags eine überzeugende Rolle auf der internationalen Bühne einnehmen? Diese Frage stellt sich angesichts der europäischen und globalen Herausforderungen mit neuer Intensität. Die Union ringt um einen gemeinsamen Auftritt, sei es im internationalen Krisenmanagement - insbesondere nach den Erfahrungen im Afghanistankonflikt - in den Kopenhagener Klimaverhandlungen oder in der internationalen Finanzkrise.

Mit dem Lissabonner Vertrag ist der Versuch verbunden, das außenpolitische Potenzial der Europäischen Union auf dem Feld der auswärtigen Beziehungen besser zu nutzen beziehungsweise überhaupt eine umfassende globale außen- und sicherheitspolitische Kultur zu entwickeln. Gleichzeitig geht die Union mit ihrer neuen Vertragsgrundlage bewusst nicht soweit, die Außen- und Sicherheitspolitik zu vergemeinschaften oder Mehrheitsentscheidungen einzuführen. ${ }^{1}$

Die außenpolitischen Gemeinsamkeiten zu bekräftigen und Differenzen zwischen den Mitgliedstaaten und den Institutionen in einem nächsten Schritt so auszutarieren, dass die Europäische Union als ein einheitlicherer Akteur agiert und wahrgenommen wird, bleibt deshalb eine Herkulesaufgabe. Insbesondere die Reform der institutionellen Grundlagen der EU-Diplomatie gestaltet sich anspruchsvoll - vorneweg der Aufbau des im Lissabonner Vertrag $^{2}$ vorgesehenen Europäischen Auswärtigen Dienstes (EAD).

Dieser Dienst soll die Hohe Vertreterin der Union für Außen- und Sicherheitspolitik Catherine Ashton in ihrer Tätigkeit unterstützen und bis dato getrennte Elemente des EU-AuBenhandelns zusammenführen. Die Einrichtung des EAD wird daher zu einer wichtigen Etappe in der Gestaltung der EU-Außenvertretung. Sie wird auch das künftige Verhältnis zur Arbeit nationaler diplomatischer Dienste entscheidend bestimmen. ${ }^{3}$

Die Schwierigkeit des vorliegenden Artikels, Stand und Perspektiven des EAD in der derzeitigen Aufbauphase zu beurteilen, rührt aus der EU-spezifischen Art, mit der dieses Inte-

* Julia Lieb, M.E.S., Forschungsassistentin, Forschungsgruppe EU-Integration, Stiftung Wissenschaft und Politik, Berlin.

Martin Kremer, M.C.L., Gastwissenschaftler, Forschungsgruppe EU-Integration, Stiftung Wissenschaft und Politik, Berlin.

Der Artikel gibt ausschließlich die persönliche Auffassung der beiden Autoren wieder.

1 Insbesondere auf britisches Drängen hin wurden zwei rechtlich unverbindliche Erklärungen verabschiedet, die die Autonomie mitgliedstaatlicher Außenpolitik festhalten. Vgl. 13. Erklärung zur Gemeinsamen Außen- und Sicherheitspolitik zum Lissabonner Vertrag, in: Amtsblatt der EU, Nr. C 83 vom 30. März 2010, S. 343; 14. Erklärung zur Gemeinsamen Außen- und Sicherheitspolitik zum Lissabonner Vertrag, in: Amtsblatt der EU, Nr. C 83 vom 30. März 2010, S. 343.

2 Art. 27 Abs. 3 Vertrag über die Europäische Union (EUV) in der konsolidierten Fassung des Lissabonner Vertrags, in: Amtsblatt der EU, Nr. C 83 vom 30. März 2010, S. 13-46. Sofern dies nicht gesondert ausgewiesen ist, bezieht sich ,EUV‘ auf die konsolidierte Fassung des Lissabonner Vertrags.

3 Dies umso mehr angesichts der Tatsache, dass die Außenminister der Mitgliedstaaten der Europäischen Union und ihre Dienste ihre mitgestaltende Rolle im Europäischen Rat teilweise abgeben mussten: Die Außenminister werden nicht mehr regelmäßig an den Europäischen Räten teilnehmen. Sie und ihre diplomatischen Dienste können künftig nur versuchen, über den Allgemeinen Rat Einfluss auf die inhaltliche Vorbereitung der Treffen der Staats- und Regierungschefs - und damit auch die außenpolitischen Leitlinien der Europäischen Union zu nehmen. 
grationsprojekt auf den Weg gebracht wurde: Einerseits wurden im Europäischen Konvent und mit dem Vertrag von Lissabon ehrgeizige Absichtserklärungen formuliert. Anderseits wurde zwar politischer Konsens erzielt, aber bewusst darauf verzichtet, Umsetzungsmaßnahmen festzulegen. Es überrascht daher nicht, dass seit dem Inkrafttreten des Lissabonner Vertrags am 1. Dezember 2009 unter erheblichem Zeitdruck eine Debatte über konkrete Ziele, Zuständigkeiten und Organisationsformen begonnen wurde und organisatorische Details sowie institutionelle und mitgliedstaatliche Einflusssicherung in den Vordergrund rückten.

Die Konturen des EAD zeichnen sich nunmehr dennoch ab. Am 21. Juni 2010 einigten sich die Hohe Vertreterin der Union für Außen- und Sicherheitspolitik und Vertreter des Europäischen Parlaments, des Rates der Europäischen Union und der Kommission über die Eckpunkte des EAD. ${ }^{4}$ Am 8. Juli 2010 hat das Europäische Parlament diesem Kompromiss mit breiter Mehrheit zugestimmt und den Weg für die endgültige Verabschiedung der Ratsentscheidung frei gemacht. ${ }^{5}$

Wo steht die Union in ihrem Bemühen, kohärente und wirksame diplomatische Strukturen auf der europäischen Ebene einzurichten? Welche Perspektiven ergeben sich daraus für die künftige Arbeit des EAD? Drei Thesen sollen die Beantwortung dieser Fragen leiten:

1. Auch wenn die Mitgliedstaaten und die Institutionen der Europäischen Union dem ,Projekt ${ }^{`}$ EAD insgesamt positiv gegenüberstanden, divergierten von Beginn an die Modelle einer gemeinsamen Außenvertretung. Zudem mangelte es an nachhaltiger Bereitschaft, die institutionelle Basis für einen kohärenten europäischen Auftritt umfassend zu reformieren.

2. Die Strategien in den bisherigen Verhandlungsrunden über den EAD waren - in einem nach wie vor zentralen Kernbereich mitgliedstaatlicher Souveränität - mehrheitlich ,defensiv`. Mitgliedstaaten und Institutionen stellten ihre Kontrolle und ihren Einfluss in den neuen diplomatischen Strukturen der Europäischen Union zunächst vor das Anliegen eines funktionsfähigen Dienstes für eine Außenpolitik , aus einem Guss‘.

3. Der Kompromiss über den Aufbau des EAD resultiert aus dieser Reformzurückhaltung auf der europäischen Ebene. Ob und wie der Dienst erfolgreich sein kann, ist sowohl abhängig von der Belastbarkeit der beschlossenen Strukturen und Verfahren als auch - und vor allem - vom Engagement der involvierten Personen. Zudem müssen wichtige Bedingungen noch gewährleistet werden, wenn dieser mehr als die Addition von 27 mitgliedstaatlichen Positionen sein soll: Sie betreffen - neben der inhaltlichen Prioritätensetzung und Ressourcenallokation - unter anderem das konstruktive Zusammenspiel im neuen institutionellen Gefüge, die Funktionsweise des Dienstes sowie eine Strategie zur Personalrekrutierung und die Herausbildung eines loyalitätsstiftenden diplomatischen Korpsgeistes in der Europäischen Union.

\section{Auf dem Weg zu den Bestimmungen des Lissabonner Vertrags}

Die allgemein gehaltenen Bestimmungen des Lissabonner Vertrags erschwerten die Verhandlungen über die institutionellen Reformen im Bereich der EU-Außenbeziehungen von Beginn an. Sie gehen auf die Arbeiten des Europäischen Konvents zurück.

Die Aufgabenstellung an den Konvent für die Reform des außenpolitischen Instrumentariums der Union gaben die Staats- und Regierungschefs der Europäischen Union in ihrer Er-

4 Stellungnahme des Europäischen Parlaments, der Hohen Vertreterin der Union für Außen- und Sicherheitspolitik, des Rates und der Kommission über den Europäischen Auswärtigen Dienst im Anschluss an ein Treffen des Quadrilogs am 21. Juni 2010 in Madrid, abrufbar unter: http://www.eu2010.es/export/sites/presidencia/ comun/descargas/Comunicados/COMUNICADO_POR_SAE_INGLES.pdf (letzter Zugriff: 05.07.2010). 
klärung von Laeken von 2001 vor. Der Konvent sollte etwa Vorschläge für „,mehr und besser koordinierte Maßnahmen bei der Bekämpfung der Krisenherde in und um Europa sowie in der übrigen Welt" und eine ,kohärentere gemeinsame Außenpolitik und Verteidigungspolitik" unterbreiten. ${ }^{6}$

Grund für diesen Auftrag war, dass Reformen - im Zuge eines parallelen Integrationsprozesses in der EU-Außenvertretung - immer dringlicher geworden waren. ${ }^{7}$ Denn seit den 1950er Jahren haben sich supranationale und intergouvernementale Politikfelder in institutioneller Hinsicht nahezu isoliert voneinander entwickelt.

Die Europäische Kommission baute parallel zur Übertragung von Kompetenzen auf die europäische Ebene einen eigenen Außendienst auf. ${ }^{8}$ Zum Zeitpunkt des Inkrafttretens des Vertrags von Lissabon verfügte sie über ein weitreichendes Netzwerk von mehr als 130 Delegationen weltweit. Ihr eigenes Selbstverständnis gründet auf einer Vielzahl außenpolitisch wirksamer Aufträge des ehemaligen Vertrags zur Gründung der Europäischen Gemeinschaft, die sich auch weiterhin in einer Ausdifferenzierung außenpolitischer Arbeitseinheiten innerhalb der Kommission widerspiegeln. ${ }^{9}$

In der aus der Europäischen Politischen Zusammenarbeit hervorgegangenen Gemeinsamen Außen- und Sicherheitspolitik (GASP) sind die Handlungsspielräume der Akteure von jeher durch das Einstimmigkeitsprinzip abgesteckt. Zwar konnte sich der frühere Hohe Vertreter für die Gemeinsame Außen- und Sicherheitspolitik, Javier Solana, in seiner Amtszeit bis 2009 mithilfe graduell ausgebauter Planungs- und Strategieeinheiten als wichtiger und ernst zu nehmender außenpolitischer Akteur der Europäischen Union etablieren. Allerdings erhielt er - wie die ihm unterstehenden EU-Sonderbeauftragten ${ }^{10}$ - einstimmig beschlossene Mandate des Rates der Europäischen Union. Er sah sich zudem in seinem Aktionsradius eingeschränkt, weil mit der außenpolitischen Troika der Europäischen Union ${ }^{11}$ und der Rolle der rotierenden Ratspräsidentschaft in der Vertretung der Union in Drittländern und bei internationalen Organisationen zusätzliche, mitunter konkurrierende Formate der GASP-Diplomatie bestanden.

Mit dem Fortschreiten des Integrationsprozesses und dem internationalen Kontext nach dem Ende des Ost-West-Konflikts war die klare Trennung zwischen Angelegenheiten der intergouvernementalen GASP und der Europäischen Gemeinschaften immer schwieriger aufrechtzuerhalten. Aufgrund der zunehmenden Überschneidung von Politikfeldern und

6 Erklärung von Laeken zur Zukunft der Europäischen Union, in: Rat der Europäischen Union: Schlussfolgerungen des Vorsitzes. Europäischer Rat (Laeken) 14. und 15. Dezember 2001, Dok. SN 300/1/01 REV 1, S. 19-26.

7 Siehe auch: Julia Lieb: Diplomatie jenseits des Staates. Eine historisch-institutionalistische Analyse des Wandels der EU-Außenvertretung, in: Benjamin von Engelhardt/Sarah H. Krieg/Isabelle Ley/Ingolf Pernice/Osvaldo Saldias (Hrsg.): Europa jenseits seiner Grenzen. Politologische, historische und juristische Perspektiven, Baden-Baden 2009, S. 169-186.

8 Siehe unter anderen: Simon Duke: Preparing for European Diplomacy, in: Journal of Common Market Studies 5/2002, S. 849-870; David Spence: The Commission's External Service, in: David Spence/Geoffrey Edwards (Hrsg.): The European Commission, London 2006, S. 396-425; Claudia Dunlea: Charles de Gaulle und das Scheitern einer europäischen Diplomatie, in: Vierteljahreshefte für Zeitgeschichte 3/2007, S. 463-484; Véronique Dimier/Mike McGeever: Diplomats without a flag. The institutionalization of the delegations of the Commission in African, Caribbean and Pacific countries, in: Journal of Common Market Studies 3/2006, S. 483505.

9 Siehe: Julia Lieb/Andreas Maurer: Europas Rolle in der Welt stärken. Optionen für ein kohärenteres Außenhandeln der Europäischen Union, Stiftung Wissenschaft und Politik: SWP-Studie 15, Berlin 2007.

10 Zur Rolle und Entwicklung der EU-Sonderbeauftragten siehe insbesondere: Cornelius Adebahr: Learning and Change in European Foreign Policy. The Case of the EU Special Representatives, Baden-Baden 2009.

11 Die Troika setzte sich gemäß dem Vertrag von Nizza (Art. 18 EUV in der Fassung des Vertrags von Nizza) aus dem Außenminister der amtierenden Ratspräsidentschaft, dem Hohen Vertreter für die Gemeinsame Außenund Sicherheitspolitik, dem Kommissar für Außenbeziehungen und gegebenenfalls dem Außenminister des künftigen Ratsvorsitzes zusammen. 
Handlungsformen im Zuge der Globalisierung und der Vielzahl von Akteuren mit teilweise stark abweichenden Präferenzen ergab sich ein - durch die Führungsschwäche der Kommission im Bereich der Außenbeziehungen verschärfter - Mangel an Kohärenz und Effizienz, der zwei Dimensionen betraf:

- die horizontale Kohärenz, das heißt die Abstimmung von Zielen, Instrumenten und Verfahren zwischen den ehemals drei Säulen der Europäischen Union;

- die vertikale Kohärenz, das heißt die Beziehungen zwischen europäischen und nationalen Handlungsebenen.

Im Konvent wurde frühzeitig die Idee des ,Doppelhuts ‘ für die EU-Außenvertretung aufgegriffen und im Sinne verbesserter außenpolitischer Kohärenz ausdifferenziert. Im Vorfeld war der ,Doppelhut' von deutscher Seite (zurückgehend auf einen gemeinsamen Vorschlag der Planungsstäbe im deutschen und französischen Außenministerium), aber auch von Vertretern des Europäischen Parlaments ins Spiel gebracht worden: Mit ihm sollten die bislang getrennten Elemente der Außenvertretung auf höchster Akteursebene zusammengeführt werden. Der Entwurfstext, der wie die Bestimmungen zum EAD nahezu identisch in den späteren Vertrag von Lissabon übernommen wurde, ${ }^{12}$ sieht das neue Amt der Hohen Vertreterin der Union für Außen- und Sicherheitspolitik vor. In diesem Posten wird das ehemalige Amt des Hohen Vertreters für die Gemeinsame Außen- und Sicherheitspolitik mit dem des Kommissars für die Außenbeziehungen der Europäischen Union fusioniert. Erste Amtsträgerin ist die Britin und ehemalige EU-Handelskommissarin Catherine Ashton.

Die Hohe Vertreterin, die vom Europäischen Rat benannt und vom Europäischen Parlament im Rahmen der Investitur der Europäischen Kommission bestätigt wird, sitzt der ebenfalls neu geschaffenen Ratsformation „Auswärtige Angelegenheiten“ vor. Als eine der Vizepräsidentinnen der Kommission ist sie gleichzeitig mit deren Zuständigkeiten im Bereich der Außenbeziehungen der Union betraut und dem Europäischen Parlament verantwortlich. ${ }^{13}$

Der Vertragstext sieht zum EAD nur vor, dass er sich aus Beamten ,,aus den einschlägigen Abteilungen des Generalsekretariat des Rates und der Kommission sowie abgeordnetem Personal der nationalen diplomatischen Dienste“ zusammensetzt. Es obliegt der Hohen Vertreterin einen Entwurf für eine Ratsentscheidung über die Organisation und Arbeitsweise des EAD zu erarbeiten, die vom Rat nach Zustimmung der Kommission und nach Anhörung des Europäischen Parlaments einstimmig verabschiedet wird. ${ }^{14}$

Weniger vage Bestimmungen zum Abschluss der Lissabonner Vertragsverhandlungen hätten überrascht, denn konkrete Reformvorschläge im Bereich der EU-Außenbeziehungen waren in der Vergangenheit immer wieder gescheitert. So wurde beispielsweise weder ein belgischer Vorstoß zur Zusammenlegung nationaler diplomatischer Strukturen noch eine deutsche Initiative zur Ausweitung der Mehrheitsentscheidung in der GASP auf europäischer Ebene aufgegriffen.

Folge der Vertragsbestimmungen war, dass die Details des künftigen institutionellen Gefüges - und damit eine Reihe politisch umstrittener und organisatorisch komplexer Fragen später in weiteren Verhandlungen zwischen den Mitgliedstaaten und den Institutionen der Europäischen Union entschieden werden mussten. Dies betrifft neben der Rolle der Hohen

12 Einzige Ausnahmen sind die oben genannten Erklärungen 13 und 14 zum Lissabonner Vertrag. Zudem wurde den Souveränitätsvorbehalten mancher Mitgliedstaaten Rechnung getragen und der neue ,Doppelhut " nicht mehr als ,Außenminister' sondern als Hohe Vertreterin der Union für Außen- und Sicherheitspolitik bezeichnet.

13 Art. 18 EUV.

14 Art. 27 Abs. 3 EUV. 
Vertreterin im Verhältnis zu anderen außenpolitischen Akteuren der Europäischen Union etwa das institutionelle Arrangement für den EAD, seine Kompetenzen und seine Funktionsweise, Personalfragen sowie die Verfahren zum Auf- und Ausbau des Dienstes.

Diese Entscheidungen mussten von Beginn an in dem weiter bestehenden Spannungsfeld zwischen supranationalen und intergouvernementalen Politikbereichen gefällt werden; denn weder der Vertrag über eine Verfassung für Europa (,Verfassungsvertrag ') noch der Vertrag von Lissabon übertragen neue außenpolitische Kompetenzen auf die europäische Ebene.

\section{Die Verhandlungsrunden zur Ausgestaltung des EAD}

Bei den Verhandlungen über den Aufbau des EAD ging es im Kern von Anfang an darum, mit welchen Zuständigkeiten der Dienst ausgestattet werden sollte. Seit der Unterzeichnung des ,Verfassungsvertrags ' durch die Staats- und Regierungschefs der Europäischen Union im Oktober 2004 fanden hierzu mehrere Verhandlungsrunden statt. Bis heute waren diese von den vornehmlich ,defensiven 'Verhandlungsstrategien der Mitgliedstaaten und Institutionen der Europäischen Union geprägt, die sich in doppelter Hinsicht bemerkbar machten.

Erstens wurden die Verhandlungen immer wieder unterbrochen - nach dem Scheitern der Referenden über den ,Verfassungsvertrag' in Frankreich und den Niederlanden im Jahr 2005 und angesichts der Notwendigkeit, das 2008 gescheiterte Referendum über den Vertrag von Lissabon in Irland 2009 zu wiederholen. Mit Verweis auf die nicht endgültig verabschiedete Vertragsänderung und die sensiblen, souveränitätsbezogenen Fragestellungen wurde es als politisch inopportun bezeichnet, die Vorarbeiten für den EAD fortzuführen. Allerdings verzichteten die Mitgliedstaaten und Institutionen der Europäischen Union auch darauf - und das Lager der EAD-Befürworter erwies sich in dieser Hinsicht als zu schwach -, sich informell und strukturiert über die künftige Gestalt des EAD auszutauschen. Dies geschah, obwohl die ersten Diskussionen verdeutlicht hatten, welchen umfassenden Beratungsbedarf es über den neuen Dienst geben würde. ${ }^{15}$

Ein Sachstandsbericht über die Ergebnisse der Konsultationen zwischen den Mitgliedstaaten und den Institutionen der Europäischen Union, den der Hohe Vertreter für die Gemeinsame Außen- und Sicherheitspolitik, Javier Solana, und Kommissionspräsident, José Manuel Barroso, dem Europäischen Rat im Juni 2005 übermittelten, formulierte denn auch nur einen dürftigen Minimalkonsens. ${ }^{16}$ Die slowenische Ratspräsidentschaft legte Anfang 2008 einen Bericht vor, der kaum über die Verhandlungsergebnisse von 2005 hinausging. ${ }^{17}$

Zweitens standen noch keine konkreten Umsetzungsmaßnahmen für die vertraglichen Bestimmungen auf der Agenda der Mitgliedstaaten und EU-Institutionen. Die Verhandlungen fokussierten daher selten auf die künftige ,Funktionsweise' des EAD. Dies änderte sich auch nicht, als nach der Sommerpause 2009 - nachdem die Ratifikationen in Tschechien und Irland abgeschlossen waren und der Lissabonner Vertrag zum 1. Dezember 2009 in Kraft treten konnte - die Vorarbeiten für den Dienst mit Nachdruck wieder aufgenommen wurden. ${ }^{18}$ Die Mitgliedstaaten und Institutionen der Europäischen Union waren wiederum

15 Siehe auch: Andreas Maurer/Sarah Reichel: Der Europäische Auswärtige Dienst. Elemente eines Drei-PhasenPlans, Stiftung Wissenschaft und Politik: SWP-Aktuell 53, Berlin 2004; Julia Lieb/Andreas Maurer: Der Europäische Auswärtige Dienst. Notwendige Vorarbeiten gegen ein böses Erwachen, Stiftung Wissenschaft und Politik: SWP-Aktuell 35, Berlin 2008.

16 Rat der Europäischen Union: Europäischer Auswärtiger Dienst. Gemeinsamer Sachstandsbericht des Generalsekretärs/Hohen Vertreters und der Kommission für den Europäischen Rat, Dok. 9956/05.

17 Rat der Europäischen Union: Sachstandsbericht des Vorsitzes an den Europäischen Rat - Vorbereitungsarbeiten im Hinblick auf das Inkrafttreten des Vertrags von Lissabon, Dok. 10650/08.

18 Siehe auch: Julia Lieb/Martin Kremer: Aufbau mit Weitsicht. Der Europäische Auswärtige Dienst als Chance für die EU-Außenpolitik, Stiftung Wissenschaft und Politik: SWP-Aktuell 2, Berlin 2010. 
hauptsächlich darauf bedacht, Macht und Einfluss in den EU-Außenbeziehungen zu wahren beziehungsweise auszubauen. Dies äußerte sich zuvorderst, in den Schwerpunkten der Beratungen im Rat der Europäischen Union. Mitgliedstaaten, Kommission und Ratssekretariat rangen von Beginn der neuen Verhandlungsphase an um Führungspositionen in den neuen diplomatischen Strukturen. Dies war mit Blick auf die Teilhabe am EAD zwar nachvollziehbar, doch blieb kaum Raum, um über vorausschauende Verfahren und eine Gesamtstrategie beim Aufbau des Dienstes nachzudenken.

Zudem zeigte sich, dass sich die neue institutionelle Architektur unter dem Lissabonner Vertrag erst einjustieren muss. Die Mitgliedstaaten und die Kommission stehen hierbei in einer wechselseitigen Abhängigkeit: Je offensiver die Kommission bislang ihren eigenen Machtbereich absteckte, desto stärker setzten sich die Mitgliedstaaten zur Wehr (und umgekehrt): Als Kommissionspräsident Barroso verkündete, einen Kommissar in seinem neuen Kollegium ab 2009 mit dem Bereich der Europäischen Nachbarschaftspolitik (ENP) zu betrauen, wurde im Rat der Europäischen Union hart um die Kontrolle der beachtlichen und außenpolitisch bedeutsamen ENP-Finanzmittel im Spannungsfeld zwischen Kommission und EAD gerungen. Als die Mitgliedstaaten mit der Ernennung des ehemaligen Kabinettschefs des Kommissionspräsidenten, João Vale de Almeida, zum EU-Botschafter in Washington überrascht wurden, forderten sie im Nachgang eine verbesserte Repräsentanz mitgliedstaatlicher Beamter auf EAD-Führungsposten sowie eine maßgebliche Rolle bei der Personalrekrutierung insgesamt ein. Im Rat der Europäischen Union hatten sie zuvor die neue Direktion Krisenmanagement und Planung (CMPD) geschaffen, in der die zivilen und militärischen Krisenreaktionsinstrumente des Rates zusammengefasst wurden. Damit entzogen die Mitgliedstaaten der Kommission mit ihren bestehenden Instrumenten für das zivile Krisenmanagement frühzeitig Möglichkeiten der Einflussnahme in diesem Bereich.

Die Frage der ,Funktionsfähigkeit' des Dienstes - etwa beim gebotenen Umfang der in den EAD zu überführenden Arbeitseinheiten aus Ratssekretariat und Kommission sowie der Vermeidung von Doppelstrukturen bei praktikablen Weisungssträngen oder den Bedingungen für eine erfolgreiche Strategie zur Personalrekrutierung - geriet mangels vorheriger Klärung dieser Machtfragen ins Hintertreffen. Neben den defensiven Verhandlungsstrategien wurde darüber hinaus deutlich, dass mit der Länge der Verhandlungen über den Aufbau des EAD die Detailfragen zunahmen. Ihre Beantwortung wurde pragmatisch und angesichts eines wachsenden Zeitdrucks auf später vertagt.

Der Verhandlungsverlauf trug wenig dazu bei, dass sich die Modelle der Mitgliedstaaten von den künftigen diplomatischen Strukturen und Verfahren der Europäischen Union einander anglichen. Die Modelle divergieren bis heute im Kern aufgrund unterschiedlicher nationaler diplomatischer und außenpolitischer Ressourcen, diplomatischer Geschichte sowie Kultur als auch spezifischen Integrationsverständnissen.

In diesem Sinne befürworten kleinere Mitgliedstaaten in der Regel den zeitnahen Aufbau des EAD. Sie sehen in einem mit Kompetenzen und Ressourcen gut aufgestellten Dienst eine Chance, ihre eigene Diplomatie zu stärken - auch wenn sie gleichzeitig oft nicht über konkrete Vorstellungen einer globalen Außen- und Sicherheitspolitik verfügen. Bei großen Mitgliedstaaten ist die Haltung oft ambivalent: Auf der einen Seite wird der Mehrwert des EAD für das Außenhandeln der Europäischen Union und nationale Verhandlungspositionen erkannt; auf der anderen Seite soll die Autonomie mitgliedstaatlicher Außenpolitik und die Rolle nationaler diplomatischer Netze nicht beschnitten werden sowie bei gemeinsamen Maßnahmen von allen Partnern außen- und sicherheitspolitische Ressourcen (zum Beispiel im Krisenmanagement) bereitgestellt werden. 
Schließlich wurde in den Verhandlungen über den EAD deutlich, dass den Mitgliedstaaten jetzt, wie auch unter dem Lissabonner Vertrag insgesamt, eine stärkere Mit-Führungsverantwortung für den Verlauf der Integration in der Union zukommt. Die Mitgliedstaaten setzen sich zwar für den weitgehenden Erhalt bestehender Strukturen der EU-Außenpolitik ein - insbesondere denen des Generalsekretariats des Rates. Gleichzeitig sind sie zunehmend mit selbstbewussten und einflussreichen EU-Institutionen konfrontiert, die nicht mehr alleine im Interesse von Sachpolitiken agieren.

Die Kommission verhinderte den weitreichenden Abzug bisheriger außenpolitischer $\mathrm{Zu}-$ ständigkeiten. Sie schuf - wie oben beschrieben - Fakten und nutzte ihren verfahrensmäßigen und institutionellen Vorsprung bei der Ausarbeitung von Beratungsvorlagen sowie bei der Umwandlung ihrer Delegationen in Delegationen der Union. Das Parlament schöpfte seine neuen Möglichkeiten voll aus und fordert seinerseits weiter reichende Mitsprache als bisher in der Außen- und Sicherheitspolitik. Bei der Ausgestaltung und der Arbeit der neuen diplomatischen Strukturen will es die ihm zur Verfügung stehenden Hebel im Entscheidungsverfahren strategisch nutzen, wie die Analyse der derzeitigen Verhandlungsphase und der Perspektiven des EAD im Folgenden zeigt.

\section{Die Konturen und Perspektiven des künftigen EAD}

Der EAD wird nach der Sommerpause 2010 seine Tätigkeit schrittweise aufnehmen können. ${ }^{19} \mathrm{Ob}$ die langfristige Handlungsfähigkeit des Dienstes durch die bisherigen Entscheidungen gewährleistet ist, wird sich im Lichte der ersten Erfahrungen zeigen..$^{20}$ Die jetzigen Beschlüsse weisen einen Kompromisscharakter auf und werden einem regelmäßigen Überprüfungsverfahren zu unterziehen sein. Gleichzeitig muss eine Reihe von Elementen für das tägliche Wirken des Dienstes erst noch bereitgestellt werden.

Den Auftakt der letzten Verhandlungsrunde bildeten Leitlinien für die Organisation und Arbeitsweise des EAD, die der Europäische Rat am 30. Oktober 2009 verabschiedete. ${ }^{21}$ Sie stellten einen wichtigen Zwischenschritt in den Verhandlungen dar. Gleichzeitig wurden zentrale Entscheidungen - etwa die Zuordnung von Befugnissen und Finanzmitteln - beim Aufbau des Dienstes zunächst vertagt. ${ }^{22}$

Ende März 2010 legte Catherine Ashton zunächst ein Organigramm des EAD und wenig später einen Entwurf für die notwendige Ratsentscheidung über den Dienst vor. ${ }^{23}$ Auch

19 Siehe unter anderem Honor Mahony: Deal on diplomatic service, in: EUObserver, 21.06.2010.

20 Beobachter gehen für die erste Phase von einem Personalbestand von circa 1.500 Mitarbeitern in der Brüsseler Zentrale und von circa 800 Diplomaten in den Delegationen der Union aus. Langfristig ist von bis zu 8.000 Mitarbeitern die Rede. Siehe unter anderem: Honor Mahony: Deal reached on diplomatic service, in: EUObserver, 21.06.2010; Andrew Rettmann: EU takes ,historic step“ in new diplomatic service, in: EUObserver, 08.07.2010; Marianne Truttmann: Der Auswärtige Dienst der EU ist startbereit, in: Neue Zürcher Zeitung, 09.07.2010.

21 Rat der Europäischen Union: Bericht des Vorsitzes an den Europäischen Rat über den Europäischen Auswärtigen Dienst, Dok. 14930/09.

22 Am 1. Dezember 2009 fällte der Rat zudem eine Entscheidung mit Auswirkungen auf die Gestalt des EAD: Nach dem Beschluss des Rates der Europäischen Union werden das Politische und Sicherheitspolitische Komitee (PSK) sowie mehrheitlich die Ratsarbeitsgruppen der GASP von Vertretern der Hohen Vertreterin geleitet, die gleichzeitig dem EAD angehören sollen. Diese Regelung soll nach einer Übergangsphase von sechs Monaten in Kraft treten. Siehe Beschluss des Rates der Europäischen Union vom 1. Dezember 2009 zur Festlegung von Maßnahmen für die Durchführung des Beschlusses des Europäischen Rates über die Ausübung des Vorsitzes im Rat und über den Vorsitz in den Vorbereitungsgremien des Rates 2009/909/EU, in: Amtsblatt der EU, Nr. L 322 vom 9. Dezember 2009, S. 28-34.

23 Proposal for a Council Decision establishing the organisation and functioning of the European External Action Service, 25.03.2010, abrufbar unter: http://eeas.europa.eu/docs/eeas_draft_decision_250310_en.pdf (letzter Zugriff: 28.06.2010). 
wenn sich der Rat der Europäischen Union bis zum 26. April 2010 schließlich überraschend schnell auf einen Kompromisstext für die Ratsentscheidung einigen konnte, erzielte er zunächst nur eine politische Vereinbarung. ${ }^{24}$ Denn der Vertrag von Lissabon sieht vor der Verabschiedung der Ratsentscheidung die Anhörung des Europäischen Parlaments vor.

Um den EAD auf den Weg zu bringen, sind nicht nur das Anhörungsverfahren zur einsetzenden Ratsentscheidung abzuschließen und ein Haushalt für den EAD aufzustellen, aus dem die Organisationsstruktur und ein Stellenplan hervorgehen. Auch das Personalstatut ${ }^{25}$ und die Haushaltsordnung ${ }^{26}$ müssen angepasst werden. Letztere unterliegen dem ordentlichen Gesetzgebungsverfahren. ${ }^{27}$ Das Parlament hatte bereits am 16. April 2010 erklärt, diese Aspekte nur im Paket zu verhandeln, und sich damit indirekt ein Vetorecht beim Aufbau des EAD durch seine gleichberechtigte Mitwirkung an der Entscheidung im Rahmen des ordentlichen Gesetzgebungsverfahrens gesichert. ${ }^{28}$

Am 21. Juni 2010 erzielte ein Quadrilog - zusammengesetzt aus der Hohen Vertreterin und Repräsentanten des Europäischen Parlaments, des Rates der Europäischen Union und der Kommission - eine politische Einigung über die letzten noch offenen Punkte beim Aufbau des EAD. Konsentiert wurden der Text der Ratsentscheidung sowie eine „Erklärung über die politische Verantwortlichkeit“ der Hohen Vertreterin gegenüber dem Europäischen Parlament und eine ,Stellungnahme über die grundlegende Organisation der Zentralverwaltung des EAD“, die die Hohe Vertreterin vor dem Plenum des Europäischen Parlaments abgeben wird. ${ }^{29}$ Am 8. Juli 2010 verabschiedete das Europäische Parlament schließlich mit breiter Mehrheit einen Bericht des deutschen Europaparlamentariers Elmar Brok ${ }^{30}$ und ermöglichte dem Rat die Verabschiedung der Entscheidung zur Einsetzung des EAD - und im Anschluss der Hohen Vertreterin, die konkrete Planung der Aufbauphase zu beginnen sowie die Einstellungsverfahren einzuleiten. Nach der Sommerpause 2010 müssen noch die Änderungen am Personalstatut und der Haushaltordnung beschlossen werden. Als mögliches ehrgeiziges Datum für die Arbeitsaufnahme des Dienstes ist vor diesem Hintergrund häufig der erste Jahrestag des Inkrafttretens des Vertrags von Lissabon, der 1. Dezember 2010, ins Spiel gebracht worden.

24 Rat der Europäischen Union: Vorschlag für einen Beschluss des Rates über die Organisation und Arbeitsweise des Europäischen Auswärtigen Dienstes - Kompromisstext des Vorsitzes, Dok. 8724/1/10.

25 Europäische Kommission: Statut der Beamten der Europäischen Gemeinschaften und Beschäftigungsbedingungen für die sonstigen Bediensteten der Europäischen Gemeinschaften, Fassung vom 01.05.2004, abrufbar unter: http://ec.europa.eu/civil_service/docs/toc100_de.pdf (letzter Zugriff: 19.06.2010).

26 Verordnung (EG, Euratom) Nr. 1605/2002 des Rates vom 25. Juni 2002 über die Haushaltsordnung für den Gesamthaushalt der Europäischen Gemeinschaften, in: Amtsblatt der EU, Nr. L 248 vom 16. September 2002, S. 1-48.

27 Bei der Änderung des Personalstatuts musste die Kommission zudem mit den Gewerkschaften verhandeln.

28 Europäisches Parlament: EU foreign service: EP links budget approval to agreement on EEAS organization, Pressemitteilung, 16.04.2010.

29 Rat der Europäischen Union: Vorschlag für einen Beschluss des Rates über die Organisation und die Arbeitsweise des Europäischen Auswärtigen Dienstes, Dok. 11507/10; Hohe Vertreterin der Union für Außen- und Sicherheitspolitik: Draft declaration by the High Representative on political accountability, Version 8 June 2010, abrufbar unter: http://www.europarl.europa.eu/meetdocs/2009_2014/documents/afet/dv/201/201007/20100706_ 02declarationpolacc_en.pdf (letzter Zugriff: 05.07.2010); Elements for a statement to be given by the High Representative in the plenary of the European Parliament on the basic organisation of the EEAS central administration, 29.06.2010, abrufbar unter: http://www.europarl.europa.eu/meetdocs/2009_2014/documents/afet/dv/201/ 201007/20100706_centraladmstat_rev_en.pdf (letzter Zugriff: 05.07.2010).

30549 Abgeordnete stimmten für und 78 gegen den Bericht, 17 enthielten sich. Siehe: Legislative Entschließung des Europäischen Parlaments vom 8. Juli 2010 zu dem Vorschlag für einen Beschluss des Rates über die Organisation und die Arbeitsweise des Europäischen Auswärtigen Dienstes (08029/2010 - C7-0090/2010 - 2010/ 0816(NLE)). 
Bei der Kompromissfindung hatten sich der Rat der Europäischen Union, die Kommission und dann auch das Europäische Parlament in mehreren Punkten aufeinander zu bewegt. Dies obwohl viele Mitgliedstaaten der Auffassung waren, dass die Ansprüche des Parlaments über den im Europäischen Konvent erzielten Konsens über Zuständigkeiten und Verfahren hinausreichten. $\mathrm{Zu}$ den Kernforderungen des Europäischen Parlaments beim Aufbau und mit Blick auf die Arbeit des EAD zählten umfassende Haushalts-, Informations- und politische Kontrollrechte. Das Europäische Parlament setzte sich außerdem für einen Dienst ein, der für ein möglichst breites Spektrum an außenpolitischen Fragestellungen zuständig ist - soweit dies keine Intergouvernementalisierung bisher vergemeinschafteter Politikfelder mit sich bringt und eine Balance zwischen zivilen und militärischen Elementen des Krisenmanagements herstellt. ${ }^{31}$

Die Verhandlungen bis zum 21. Juni 2010 brachten folgende Eckpunkte für den EAD hervor, die im Entwurf der Ratsentscheidung sowie der Erklärung und der Stellungnahme festgehalten sind und außerdem in die Änderungen des Personalstatuts und der Haushaltsordnung einfließen sollen:

Rechtsstatus: Der EAD wird eine Dienststelle , sui generis' zwischen Kommission und Ratssekretariat und unter der Verantwortung der Hohen Vertreterin. Die Mitgliedstaaten haben sich von Anfang an gegen eine Ansiedlung der bisherigen Krisenmanagementstrukturen - und der GASP im Allgemeinen - bei der Kommission verwahrt. Die Kommission hat den Kompromiss frühzeitig mitgetragen, verhinderte aber wiederum erfolgreich einen weitreichenden Abzug ihrer eigenen außenpolitischen Kompetenzfelder in den EAD.

Das unter dem Lissabonner Vertrag machtbewusstere Europäische Parlament hat lange allerdings zumeist im Sinne einer Verhandlungsposition und um dem Rat Zugeständnisse abzuringen - die Eingliederung des EAD als ,eigenständige Dienststelle in organisatorischer und haushaltstechnischer Hinsicht in die Verwaltungsstrukturen der Kommission“ verlangt, um ein Maximum an Kontrollrechten ableiten zu können. Das Parlament konnte sich mit dieser Forderung nicht durchsetzen, erkämpfte jedoch eine Reihe von Zugeständnissen: Insbesondere der Haushalt des EAD wird voll der Kontrolle des Europäischen Parlaments unterliegen. Außerdem wurden dem Parlament etwa in der Erklärung über die politische Verantwortlichkeit der Hohen Vertreterin neue Rechte beim Zugang zu Informationen zugesichert.

Kompetenzen, Struktur und Finanzinstrumente: Im Zuge der Kompetenzübertragung in den Außenbeziehungen, der Entwicklungszusammenarbeit und dem Krisenmanagement an den EAD setzte sich nicht zuletzt auf Betreiben der Kommission und des Europäischen Parlaments die Lesart des Vertrags durch, dass der Kommission weiterhin breite Zuständigkeiten und Aufträge - etwa in der Handels-, aber auch in der Nachbarschafts- und Erweiterungs- oder der Klimapolitik - zukommen. Die Strukturen des EAD und die grob definierten Verbindungen zur Kommission tragen dieser Tatsache Rechnung.

Die Zentralverwaltung des EAD wird aus geografisch, multilateral und thematisch ausgerichteten Einheiten bestehen. Sie sollen mit den betreffenden Diensten der Kommission und des Generalsekretariats des Rates zusammenarbeiten und neben den bilateralen Beziehun-

31 Grundlage der Forderungen und Arbeiten des Europäischen Parlaments war ein Arbeitspapier der beiden Berichterstatter zum EAD. Elmar Brok/Guy Verhofstadt: Proposal for the establishment of the EEAS, Arbeitspapier, aktualisierte Version, 20.04.2010, abrufbar unter: http://www.europarl.europa.eu/meetdocs/2009_2014/ documents/afet/dv/201/201006/20100602_finalproposal_en.pdf (letzter Zugriff: 05.07.2010). Auch andere Fraktion wie die S\&D-Fraktion legten interne Vermerke und Positionspapiere vor. Vgl. Bernhard Rapkay/Jutta Haug: EuropaThemen-direkt: Europäischer Auswärtiger Dienst, Mai 2010, abrufbar unter: http://spdnet.sozi.info/nrw/dortmund/brapkay/dl/EuropaThemenDirekt_EAD.pdf (letzter Zugriff: 05.07.2010). 
gen der Europäischen Union zu allen Drittstaaten die GASP, einschließlich der Gemeinsamen Sicherheits- und Verteidigungspolitik (GSVP), abdecken.

Komplex wird sich vor diesem Hintergrund etwa die Vergabe von Finanzmitteln ${ }^{32}$ gestalten. Entscheidungen sollen in fünf sogenannten ,Programmierungsphasen ' teils im EAD (in der konzeptionellen Anfangsphase), teils auch in der Kommission (in der entscheidenden Schlussphase) vorbereitet und implementiert werden. Neben der Hohen Vertreterin werden zudem einzelne Kommissare - insbesondere die Kommissare für Erweiterung und Europäische Nachbarschaftspolitik sowie Entwicklung - eine wichtige Rolle bei der Vergabe der Mittel einnehmen. Wie die Akteure und Einheiten zusammenspielen und ob die Abläufe ohne Reibungsverluste von statten gehen können, muss sich noch erweisen.

Erklärte Absicht von Mitgliedstaaten und Institutionen der Europäischen Union ist es seit Langem, das zivile und militärische Kriseninstrumentarium der Union im Sinne einer, vernetzten Sicherheit" und ganzheitlichen Krisen- und Konfliktprävention zur Wirkung zu bringen. Bei den Verhandlungen über den EAD lehnten die Mitgliedstaaten Änderungen an den bestehenden zivilen und insbesondere militärischen Krisenreaktionsstrukturen allerdings ab. Die Krisenmanagementstrukturen der GSVP werden Teil des EAD, innerhalb des Dienstes aber getrennt von den sonstigen Aufgabenbereichen und unter der direkten Verantwortung der Hohen Vertreterin angesiedelt. ${ }^{33}$ Gleichzeitig verpflichtet sich die Hohe Vertreterin in ihrer Stellungnahme über die grundlegende Organisation der Zentralverwaltung des EAD gegenüber dem Europäischen Parlament die Krisenmanagementstrukturen in das Tagesgeschäft des Dienstes einzubinden.

Ein Streitpunkt mit dem Europäischen Parlament, der Auswirkungen auf die Struktur des EAD hat, war die Vertretung der Hohen Vertreterin im Plenum des Europäischen Parlaments. Teile des Parlaments forderten eine politische Vertretung und stellten sich gegen die Vertretung durch einen EAD-Generalsekretär aus der EAD-Verwaltung. Die Vertretung kann daher künftig von einem Außenminister der rotierenden Ratspräsidentschaft oder der Triopräsidentschaft oder einem Kommissar übernommen werden. ${ }^{34} \mathrm{Im}$ EAD soll es einen ,exekutiven' Generalsekretär und zwei Stellvertreter geben. Insofern setzte sich das französische Modell von Generalsekretären durch, wie es etwa im Quai d'Orsay, dem französischen Außenministerium, praktiziert wird. Die Führungsstruktur des EAD ist auf diese Weise auf das Management und die inhaltliche Orientierung des Dienstes ausgerichtet. Auf Betreiben des Europäischen Parlaments hin soll außerdem einer der sieben Generaldirektoren des Dienstes speziell für Haushalts- und Verwaltungsaspekte zuständig sein.

Die ehemaligen Delegationen der Kommission sind mit Inkrafttreten des Lissabonner Vertrags schon in Delegationen der Union umbenannt worden. Sie haben an mehreren Standorten bereits die Rolle der rotierenden Ratspräsidentschaft übernommen ${ }^{35}$ und sollen integraler Bestandteil des EAD werden. Ein Stufenplan für die umfassende Umwandlung des Delegationsnetzwerks für die Tätigkeiten des neuen Außendienstes ist bislang nicht vorgestellt worden. Auch die Vertretung der Europäischen Union bei internationalen Organisationen und in internationalen Verhandlungen ist noch nicht abschließend geregelt. Zum ei-

32 Darunter fallen die Instrumente für Entwicklungszusammenarbeit, der Europäische Entwicklungsfonds, das Instrument für Demokratie und Menschenrechte, das Nachbarschaftsinstrument, das Instrument für Zusammenarbeit mit Industrieländern, das Instrument für Nukleare Sicherheit sowie das Stabilitätsinstrument.

33 Für die Trennung setzte sich insbesondere Frankreich ein, erkennbar mit der Zielsetzung EU-eigene Führungsfähigkeiten zu sichern, nicht aber die Fähigkeiten auf dem Gebiet der Konfliktprävention, der Krisenreaktion und der Konfliktnachsorge organisatorisch zu vereinheitlichen.

34 Dies wurde durch die Erklärung über die politische Verantwortlichkeit der Hohen Vertreterin vereinbart.

35 Siehe unter anderem: Andrew Rettman: EU commission ,embassies“ granted new powers, in: EUObserver, 21.01.2010. 
nen besteht noch keine Einigung darüber, wie die Koordinierungsfunktionen in verschiedenen Politikfeldern zwischen dem EAD und der rotierenden Ratspräsidentschaft aufgeteilt werden sollen. Zum anderen ist die Vergabe von Verhandlungsmandaten und von Kompetenzen zum Abschluss internationaler Verträge noch nicht geklärt. ${ }^{36}$

Die Weisungsstränge zwischen Brüssel und den Delegationen sind bereits grob vorgezeichnet: Die Weisungen an die Delegationen sollen über den EAD erteilt werden. Im Zuständigkeitsbereich der Kommission kann diese auch direkte Weisungen an die Delegationen erteilen. Der EAD erhält in diesen Fällen eine Kopie der Weisungen.

Personalfragen: Wichtige Entscheidungen mussten in den Verhandlungen auch in Personalfragen erzielt werden. Der Hohen Vertreterin wird die Personalverantwortung für alle Mitarbeiter des EAD zukommen. Bei der Änderung des Personalstatuts ging es insbesondere auch darum, eine Gleichbehandlung dieses Personals unterschiedlicher Herkunft zu gewährleisten. Bis dato waren entsandte mitgliedstaatliche Beamte nicht mit Angehörigen der Kommission oder des Ratssekretariats gleichgestellt. Letztere verfügten über weiter reichende Rechte und Kompetenzen - zum Beispiel bei der Verwaltung von Finanzmitteln und erhielten umfassendere Unterstützungsleistungen bei der Aufnahme einer Tätigkeit im Ausland.

Die Mitgliedstaaten waren vom Beginn der Verhandlungen an zudem auf eine angemessene personelle Präsenz im EAD bedacht - vereinbart wurde, dass der Anteil mitgliedstaatlicher Diplomaten auf allen Hierarchieebenen des Dienstes ein Drittel ausmachen und rasch mit der Ausschreibung der ersten Delegationsleiter-, Leitungs- und Referentenposten begonnen werden soll. Die Hohe Vertreterin verpflichte sich außerdem, eine ,roadmap" vorzulegen, wie dieses Drittel bis zum Jahr 2013 erreicht werden kann. Gleichzeitig sollen durch die Entsendung von Personal in den EAD keine Mehrkosten entstehen - für die Einrichtung des Dienstes wurde das Prinzip der Haushaltsneutralität festgeschrieben.

Auch wenn die Kommission dies selbst nicht mehr forderte, setze sich das Europäische Parlament - auch im Sinne der eigenen Kontrollmöglichkeiten des EAD - dafür ein, dass der Anteil des Kommissionspersonals im Dienst 50 Prozent betragen soll. Da das Ratssekretariat über vergleichsweise wenige Beschäftigte im außenpolitischen Bereich verfügt, wird der Anteil der Kommission jedoch ohnehin weit über einem Drittel des Gesamtpersonals liegen - unabhängig von eingeführten Schwellenwerten.

Das Auswahlverfahren für das Personal des EAD über Auswahlausschüsse wird von der Hohen Vertreterin ausgearbeitet und eingeleitet. Bei der Ernennung der Delegationsleiter, die mit Blick auf die Präsenz und den Einfluss im Dienst für die Mitgliedstaaten wie die EUInstitutionen sehr wichtig ist, trifft die Hohe Vertreterin ihre Entscheidung auf der Basis einer , shortlist", der die Kommission im Vorfeld zustimmen muss. Dem Europäischen Parlament wurde zudem das Recht eingeräumt, neu benannte Delegationsleiter bei strategisch wichtigen Partnern oder internationalen Organisationen sowie EU-Sonderbeauftragte in die Parlamentsausschüsse einzuladen.

Wenn der Dienst seine Tätigkeit aufnimmt wird sich zeigen, wo der jetzige Kompromiss nicht detailliert genug oder funktionsfähig ist und angesichts der Erfahrungen in Brüssel, aber auch in Drittstaaten, zeitnah angepasst werden muss. Folgende vier Themenfelder werden von zentraler Bedeutung sein, wenn es darum geht, die richtige Balance zwischen einem ausreichenden Maß an Regelung und einer hinreichenden Dosis an Flexibilität für den EAD zu finden:

36 Seit dem Inkrafttreten des Lissabonner Vertrags kam es hier mehrfach zu Unstimmigkeiten. Siehe etwa: European Voice: Institutions locked in battle over external representation 03.06.2010; European Voice: Dispute leaves EU without voice in mercury talks, 10.06.2010. 
Konstruktives Zusammenspiel im institutionellen Gefüge: Gerät der EAD ,zwischen die Fronten" oder wird er das breite Spektrum an Politikfeldern mit Außendimension besser koordinieren können? Wird die Hohe Vertreterin konstruktiv mit dem außenpolitisch immer stärker agierenden Präsidenten des Europäischen Rates, dem Kommissionspräsidenten und den Kommissaren, den Vertretern der rotierenden Ratspräsidentschaft ${ }^{37}$ sowie dem Europäischen Parlament zusammenarbeiten können oder wird das Konfliktpotenzial auf der neuen Vertragsgrundlage zunehmen? Kann der Vorteil genutzt werden, dass die Aufgaben der rotierenden Ratspräsidentschaft - der Vorsitz im Rat für „Auswärtige Angelegenheiten“, die Vertretung der Union im politischen Dialog mit Drittstaaten sowie gegenüber dem Europäischen Parlament - auf die Hohe Vertreterin übertragen wurden und die Präsidentschaft in vielen außenpolitischen Ratsarbeitsgruppen von Mitgliedern des EAD abgelöst wird? Welche Aufgabenteilung kann es in Drittstaaten und bei internationalen Organisationen zwischen den Delegationen der Union und der rotierenden Ratspräsidentschaft geben, wenn letztere in einer Reihe von Politikfeldern weiterhin eine Rolle spielt?

Funktionsfähigkeit des Dienstes: Werden sich die außenpolitischen Dienststellen in Brüssel ausreichend konsultieren, politisch koordinieren und reibungslose und zielgerichtete Verfahren unter Beteiligung der Hohen Vertreterin, der Leitungsebene des EAD sowie den relevanten Kommissaren gewährleisten? Werden mit dem EAD demzufolge Politikentscheidungsprozesse und deren Umsetzung besser koordiniert sowie Synergien (langfristig beispielsweise auch im Konsularbereich) möglich gemacht?

Erweisen sich die Zuständigkeitsabgrenzungen in den vielen geteilten Verantwortungsbereichen als belastbar? Bieten die Weisungsstränge praktikable und eindeutige Handlungsanweisungen für das EAD-Personal in Drittstaaten und bei internationalen Organisationen? Können die divergierenden und teils veralteten Systeme der Berichterstattung in den EUAußenbeziehungen für den EAD vereinheitlicht und modernisiert werden?

Strategie zur Personalrekrutierung und Herausbildung eines diplomatischen Korpsgeistes: Wird der EAD zu einem „Sozialisationsraum“, 38 in dem sich ein spezifischer europäischer diplomatischer Korpsgeist entwickeln kann?

Kann das Personal für den EAD bis hin zur Führungsebene von Anfang an so rekrutiert und integriert werden, dass kein Nachteil für die personell erst noch , nachziehenden “ - Mitgliedstaaten entsteht? Wie kann eine geografische und politische Balance beim EAD-Personal zwischen großen und kleinen, , alten' und ,neuen' Mitgliedstaaten hergestellt werden? ${ }^{39}$ Können das Rotationsprinzip und die Rückkehr aus dem EAD so gestaltet werden, dass die Tätigkeit in diesem Dienst nicht zu einem Nachteil, sondern zu einer interessanten Karriereoption wird und somit zugleich qualifiziertes Personal anzieht? Könnte die personelle Vernetzung auch dadurch verstärkt werden, dass Angehörige aus der Kommission und dem Ratssekretariat in die nationalen diplomatischen Dienste entsandt werden?

37 Die rotierende Ratspräsidentschaft wird in den Ratsarbeitsgruppen mit außen- und sicherheitspolitischen Bezügen den Vorsitz an den EAD abgeben. Sie wird aber in einer Reihe von Politikbereichen mit außenpolitischen Bezügen weiterhin die Sitzungen leiten und damit in den EU-Außenbeziehungen insgesamt von Bedeutung sein. Vgl. Rat der Europäischen Union: Bericht des Vorsitzes über den Europäischen Auswärtigen Dienst, 2009.

38 Stefani Weiss, Europas Auswärtiger Dienst. Viel Lärm um nichts, Bertelsmann Stiftung: spotlight europe 5/2010.

39 Eine Reihe ,neuer' EU-Mitgliedstaaten machte sich für eine geografische Balance im EAD besonders stark. Siehe unter anderem: EUObserver: New EU states make bid for diplomatic cloud, 10.03.2010. Allerdings werden Quotenregelungen im Allgemeinen als wenig praktikabel und teils problematisch für die Arbeit des EAD erachtet, da hochqualifiziertes und gerade für die Auslandsposten jeweils geeignetes Personal rekrutiert werden sollte. 
Wie können bestehende außen- und sicherheitspolitische Ausbildungsmaßnahmen der Europäischen Union im Sinne einer umfassenden Ausbildung für die Tätigkeiten im EAD, aber auch in verbundenen außenpolitischen Einheiten, ausgestaltet werden. ${ }^{40}$

Überprüfungsverfahren: Im Rahmen des EAD-Aufbaus ist für 2011 ein Bericht der Hohen Vertreterin über die Funktionsweise des EAD vorgesehen. 2013 soll zudem eine Überprüfung der Ratsentscheidung zum EAD eingeleitet und bis spätestens Anfang 2014 abgeschlossen werden: Wie können aus den Erfahrungen der ersten Aufbauphase heraus Kriterien und Verfahren für eine zielgerichtete Anpassung des Aufbaus und der Arbeitsweise des Dienstes bestimmt werden?

\section{,Legitimation durch Erfolg6}

Die internationale ,Schonzeit ${ }^{41}$ für die Europäische Union ist abgelaufen: Sie kann sich im Zuge der Umsetzung des Lissabonner Vertrags nicht weiterhin vornehmlich mit sich selbst beschäftigen und keine belastbaren institutionellen Grundlagen für eine kohärente $\mathrm{Au}-$ Benvertretung schaffen. Die Mitgliedstaaten der Europäischen Union stehen mit in der Verantwortung: Sie müssen die Hohe Vertreterin in ihrer Tätigkeit, aber auch bei der Fortentwicklung der Strukturen und Verfahren des EAD unterstützen. Bislang ist diese mitgliedstaatliche Unterstützung uneinheitlich. Während zum Beispiel Frankreich in den Regierungsverhandlungen dem EAD geschickt seinen Stempel aufdrückte (etwa bei der Organisationsstruktur) waren insbesondere deutsche Parlamentarier in den Ausschüssen des Europäischen Parlaments federführend. Der britische Beitrag zur konkreten Ausgestaltung des EAD jenseits personeller Aspekte fiel demgegenüber - vor allem seit dem Antritt der neuen Regierung im Mai 2010 - gering aus.

Mit dem EAD wird nicht nur ein Dienst, sui generis' geschaffen, der sich in Krisen zu bewähren hat und der einen Mehrwert gegenüber der Diplomatie der Mitgliedstaaten erbringen muss. Er bedingt auch - neben der Chance einer stärkeren demokratischen Mitgestaltung durch das Europäische Parlament und die nationalen Parlamente - eine Entscheidung über die Inhalte und Schwerpunkte der künftigen EU-Außenbeziehungen. Die Hohe Vertreterin, die EU-Institutionen und die Mitgliedstaaten sind gut beraten, auf eine anspruchsvollere außen- und sicherheitspolitische Prioritätensetzung hinzuwirken - etwa in einem ,mission statement" oder einem Mehrjahresprogramm. Diese muss den Fähigkeiten der Union gerecht werden, die dafür notwendigen Ressourcen bereithalten und in den Verhandlungen zum neuen Finanzrahmen 2014-2020 berücksichtigt werden. In diesem Sinne muss das Hauptaugenmerk zum einen der direkten Nachbarschaft der Europäischen Union gelten, zumal die Union an ihrem Umgang mit ihren Nachbarn gemessen wird und für diesen Bereich bereits umfangreiche außenpolitische und diplomatische Instrumente und Erfahrungen bestehen. Zum anderen ist die Europäische Union unmittelbar in ihren strategischen Partnerschaften mit den USA und den aufsteigenden Weltmächten gefordert, wenn sie ihrer Akteursrolle auf der internationalen Ebene gerecht werden will.

Weitere institutionelle Machtkämpfe sollten die Hohe Vertreterin und die EU-Institutionen - auch im eigenen Interesse - vermeiden und den EAD vielmehr zu einem Dreh- und Angelpunkt der EU-Außenbeziehungen werden lassen. Die Außenpolitik der Europäischen

40 Bestehende Ausbildungsmaßnahmen sind etwa das European Diplomatic Programme (EDP), das Europäische Sicherheits- und Verteidigungskolleg (ESVK) oder die European Group on Training (EGT) für das zivile Krisenmanagement. Siehe hierzu: Julia Lieb: Consolidating civilian and military training for crisis management: taking stock of EU initiatives, Standard Briefing für das Europäische Parlament, April 2010.

41 Volker Perthes: Die Europäische Union in der Welt. Die Sicht der anderen und die Aufgaben der nahen Zukunft, in: Universitas 4/2010, S. 341-349. 
Union könnte so an Legitimation gewinnen: Legitimation durch konstruktive Beiträge im vernetzten Krisenmanagement sowie in der regionalen und globalen Ordnungspolitik. Überzeugen muss die Europäische Union nach außen wie nach innen ebenso dadurch, dass sie in ihrem Außenhandeln der zunehmenden Überschneidung von Politikfeldern gerecht wird. Der EAD sollte hierfür nach und nach eine Verbindung klassischer Außen- und Sicherheitspolitik mit den wichtigen internationalen Fragestellungen des 21. Jahrhunderts, namentlich der Klimapolitik, der Energieversorgungssicherheit, der Armutsbekämpfung oder den Menschenrechten gewährleisten - auch wenn er für letztere teils erst noch Zuständigkeit im strengen Sinne erhalten müsste.

\section{Der Vertrag von Lissabon ist in Kraft}

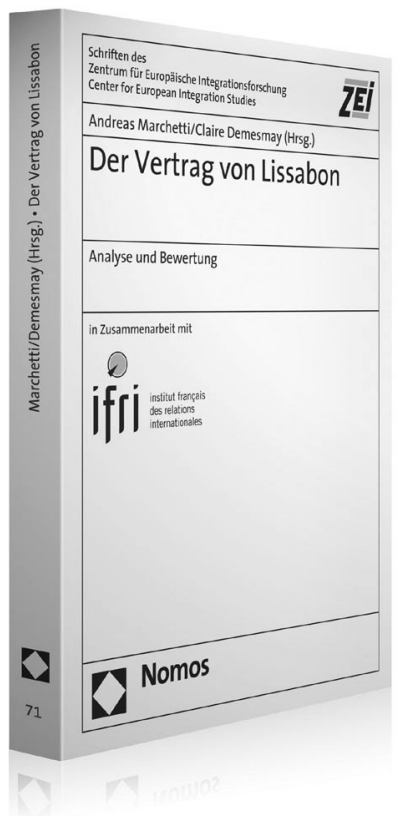

Der Vertrag von Lissabon

Analyse und Bewertung

Herausgegeben von Andreas Marchetti und Claire Demesmay

2010, 289 S., geb., 49,-€,

ISBN 978-3-8329-3676-1

(Schriften des Zentrum für Europäische Integrationsforschung (ZEI), Bd. 71)

Mit dem Vertrag von Lissabon beschließt die EU ihren langjährigen Reformprozess. Die Autoren kommentieren das neue europäische Primärrecht in interdisziplinärer Perspektive. Sie bewerten den Vertrag im Vergleich zum Vertrag von Nizza als rechtlichem Referenzpunkt und dem gescheiterten europäischen Verfassungsvertrag als politischem Orientierungspunkt. 